\title{
Inventario Psicológico de Ejecución Deportiva para Árbitros (IPEDA): Adaptación y Propiedades de Medida
}

\author{
Sport Performance Psychological Inventory for Referees (IPEDA): Adaptation and \\ Measurement Properties
}

\author{
Juan Antonio Campos-Salinas ${ }^{1}$, Rosendo Berengüi ${ }^{2}$ y Antonio Hernández-Mendo ${ }^{3}$
}

\begin{abstract}
Resumen
El estudio presenta la adaptación y propiedades del Inventario Psicológico de Ejecución Deportiva (IPED) al arbitraje deportivo (IPEDA) que evalúa las habilidades psicológicas del árbitro. El cuestionario está compuesto de 42 ítems divididos en siete escalas: autoconfianza, control de afrontamiento negativo, control atencional, control visual e imaginativo, nivel motivacional, control de afrontamiento positivo y control actitudinal. La muestra estuvo compuesta por 174 árbitros de fútbol, con una media para la edad de 22.41 años (D.T.=4.37). El cuestionario presenta adecuada consistencia interna de sus escalas, los índices de ajuste (NFI, CFI, GFI y AGFI) son superiores a .90, y los de error (RMSEA, RMR y SRMR) cercanos a .08. Además, mediante análisis de generalizabilidad el resultado es que la muestra es normal, lineal y homocedástica, con niveles altos de fiabilidad $\left(e^{2}=1\right)$ y generalizabilidad $(\Phi=1)$. Estos resultados indican que el IPEDA es un instrumento válido y preciso, capaz de ofrecer resultados fiables y generalizables.
\end{abstract}

Palabras clave: árbitro, arbitraje deportivo, evaluación, rendimiento, psicometría

\begin{abstract}
The study presents the adaptation and properties of the Psychological Inventory of Sports Performance (IPED to sports referee (IPEDA), which evaluates the psychological skills of the referee. The questionnaire is composed of 42 items divided into seven scales: self-confidence, negative coping control, attention control, visual and imaginative control, motivational level, positive coping control and attitudinal control. The sample consisted of 174 football referees, with a mean age of 22.41 years ( $\mathrm{SD}=4.37$ ). The scales have adequate internal consistency, the adjustment indexes (NFI, CFI, GFI and AGFI) are higher than .90, and the error rates (RMSEA, RMR and SRMR) around .08. In addition, through generalizability analysis the result is that sample is normal, linear and homoscedastic, with high levels of reliability $\left(e^{2}=1\right)$ and generalizability $(\Phi=1)$. These results indicate that the IPEDA is a valid and accurate instrument capable of providing reliable and generalizable results.
\end{abstract}

Keywords: referee, sports officiating, assessment, sports performance, psychometrics

\footnotetext{
${ }^{1}$ Doctor en Ciencias del Deporte. Universidad Católica de Murcia. Campus de los Jerónimos, s/n 30107 Murcia, España. Tel.: 968278181. Correo: j_campos6@hotmail.com

${ }^{2}$ Doctor en Psicología. Facultad de Ciencias Sociales y de la Comunicación. Universidad Católica de Murcia. Campus de los Jerónimos, s/n 30107 Murcia, España. Tel.: 968278181. Correo: rberengui@ucam.edu

${ }^{3}$ Doctor en Psicología. Facultad de Psicología. Universidad de Málaga. Campus de Teatinos, s/n. 29071 Málaga, España. Correo: mendo@uma.es

Revista Iberoamericana de Diagnóstico y Evaluación - e Avaliação Psicológica. RIDEP · No53 · Vol.4 · 141-152 · 2019

ISSN: 1135-3848 print /2183-6051online
} 


\section{Introducción}

La figura del árbitro es imprescindible en cualquier deporte, pues es la encargada de velar por el cumplimiento del reglamento de competición. Sus decisiones pueden alterar el desarrollo del partido o la competición (Guillén \& Jiménez, 2001), y junto a jugadores y técnicos, ha sido y es partícipe del desarrollo y evolución del deporte. Es preciso contemplar al árbitro como cualquier otro deportista, que realiza un trabajo de preparación y mejora para alcanzar sus propios objetivos propuestos, y principalmente, para poder cumplir de forma óptima con su función, razón por la cual se hacen necesarios estudios que ayuden a conocer sus puntos fuertes y aquellos precisos de mejora, con vistas a optimizar su formación, preparación y rendimiento.

Los factores que influyen en el rendimiento del arbitraje, y sobre los cuales deben ser canalizadas las investigaciones, son la preparación física, el entrenamiento de la táctica, la técnica y el entrenamiento psicológico (González-Oya \& Díaz, 2007). La preparación física busca mejorar las cualidades físicas influyentes en la práctica (Bueno \& Da Silva, 2015), mientras que para la mejora de la técnica y la táctica se deberá conocer el reglamento a la perfección, sus interpretaciones y continuas modificaciones, y estar al día de las estrategias tácticas para la óptima gestión de los partidos (Campos-Salinas, 2017). En cuanto al entrenamiento psicológico, suele ser usualmente desatendido en el entrenamiento habitual de los árbitros (Giske, Haugen, \& Johansen, 2016), y no se dispone en la actualidad de unas directrices claras y definidas para el mismo, a pesar de existir evidencias sobre la influencia de factores como el estrés, la concentración, la ansiedad o la motivación, entre otros, sobre su rendimiento (Hancock, Rix-Lievre, \& Côté, 2015). A pesar de que el nivel físico, técnico y táctico está aumentando considerablemente en los últimos años (Casajús \& González-Aguero, 2015), es limitada la atención que reciben las variables psicológicas en el arbitraje, situación que contrasta con la enorme presión que reciben los árbitros por parte del entorno, tanto en los partidos como fuera de los mismos, constituyendo una circunstancia que alerta sobre la necesidad de una preparación psicológica acorde a sus necesidades.

Es por ello, que con el estudio y determinación de las diferentes habilidades y características psicológicas que influyen en el rendimiento del árbitro, se podrá determinar cuáles deben ser entrenadas y/o potenciadas. Posiblemente, el método más sencillo para obtener esos datos sea a través de cuestionarios elaborados para medir las diferentes variables psicológicas implicadas en el rendimiento. Un ejemplo en el deporte es el Inventario Psicológico de Ejecución Deportiva (IPED), de HernándezMendo (2006) y Hernández-Mendo, MoralesSánchez y Peñalver (2014). El IPED es la adaptación del Psychological Performance Inventory (PPI) de Loehr (1986). El trabajo de Loehr parte del constructo mental toughness (dureza mental), utilizado para describir la habilidad del deportista para mantenerse de forma consistente en un estado ideal de rendimiento durante el transcurso de la competición (Loehr, 1982, 1986), sin preocuparse por las circunstancias competitivas, y lograr ese nivel de rendimiento requiere que el individuo sea física, mental, y emocionalmente fuerte o tenaz. La dureza mental del deportista estaría integrada según Loehr (1995) por tres dimensiones: dimensión física (estar bien preparado y rendir de forma enérgica), dimensión mental (habilidad para crear un estado óptimo de rendimiento, acceso a emociones potenciadoras, $\mathrm{y}$ afrontamiento), $\mathrm{y}$ dimensión emocional (flexibilidad, responsibidad, fuerza y resiliencia). A partir de esos planteamientos Loehr (1986) construye su instrumento de evaluación, el PPI.

El IPED ha sido utilizado para valorar las habilidades psicológicas de deportistas de un amplia variedad de modalidades, tales como la lucha olímpica (Berengüí, López-Gullón, Garcés de los Fayos, \& Almarcha, 2011; López-Gullón et al., 2011; López-Gullón et al., 2012), fútbol (Hernández-Mendo et al., 2014; Izquierdo et al., 2006), piragüismo (Gómez-López, GraneroGallegos, \& Isorna, 2013; Hernández-Mendo et al., 2014), taekwondo (Álvarez, Estevan, Falco, Hernández-Mendo, \& Castillo, 2014; Berengüí, Garcés de Los Fayos, \& Hidalgo-Montesinos, 2013), atletismo (Hernández-Mendo et al., 2014; Pablos, 2005 citado en López-Gullón et al., 2011) 
o triatlón (Hernández-Mendo et al., 2014; LópezCazorla, Hernández-Mendo, Reigal, \& MoralesSánchez, 2015). Sin embargo, a pesar de su capacidad para medir de forma rápida y sencilla diferentes habilidades psicológicas, el IPED no ha sido empleado en el arbitraje.

Existen trabajos similares al presente que han presentado las propiedades psicométricas del instrumento. En el estudio original del IPED (Hernández-Mendo, 2006) la fiabilidad (alfa de Cronbach) de las escalas oscila entre .69 y .74, entre .69 y .75 en fiabilidad compuesta, y presenta índices de ajuste adecuados. También, en la replicación de las propiedades psicométricas del IPED por parte de Hernández-Mendo et al. (2014), la fiabilidad compuesta oscila entre .71 y .79, y en la adaptación al contexto deportivo argentino de Raimundi, Reigal y Hernández-Mendo (2016), la fiabilidad $(\alpha)$ de las escalas se sitúa entre .67 y .76 , y la fiabilidad compuesta entre .70 y .80 . Ambos trabajos (Hernández-Mendo et al., 2014; Raimundi et al., 2016) obtienen resultados satisfactorios en índices de bondad de ajuste y error, así como en fiabilidad compuesta, varianza media extractada, validez convergente y validez discriminante.

Por su parte, el único análisis de generalizabilidad que se realiza con el IPED es el presentado por Hernández-Mendo (2006), presenta una generalizabilidad satisfactoria, que anima a futuras investigaciones a realizar dicho análisis para poder generalizar los resultados en distintas modalidades deportivas, como en este caso.

El IPED se muestra como un instrumento con adecuadas propiedades psicométricas, y su validación permitiría disponer de una herramienta útil para evaluar las habilidades psicológicas del árbitro, y así poder obtener un perfil de puntos fuertes y necesarios de mejora, y que contribuya a establecer un trabajo específico que permita optimizar su rendimiento.

Por todo ello, el objetivo de este trabajo fue la adaptación del Inventario Psicológico de Ejecución Deportiva para su empleo en el arbitraje deportivo.

\section{Método}

\section{Participantes}

Participaron en el estudio 174 árbitros y árbitros asistentes de fútbol, 167 masculinos (96\%) y 7 femeninos (4\%), con edades comprendidas entre los 13 y los 40 años, y una media de 22.41 años (D.T.= 4.37), pertenecientes a los comités territoriales de Murcia, Tenerife y Cádiz (España). Se establecieron como criterios de inclusión en el estudio estar en activo en sus comités y aptos para la práctica del arbitraje, para lo cual hay que superar las pruebas periódicas físicas y técnicas establecidas por los comités. Los participantes tuvieron que firmar una hoja de consentimiento previo antes de la realización del cuestionario, ofreciendo su conformidad para participar en este estudio. Además, esta investigación se llevó a cabo de conformidad con la Declaración de Helsinki sobre Investigaciones Biomédicas en Humanos (18th Medical Assembly, 1964; revisada en 2008 en Seúl), y las marcadas por la Ley Orgánica 15/1999, de 13 de Diciembre, de Protección de Datos de Carácter Personal.

\section{Instrumento}

El instrumento usado fue el Inventario Psicológico de Ejecución Deportiva para Árbitros (IPEDA). Se trata de una adaptación del IPED de Hernández-Mendo (2006), el cual a su vez es la versión española de la escala de Loehr (1990) Psychological Performance Inventory (PPI). Está compuesto de 42 ítems, divididos en siete subescalas con seis ítems cada una. Cada ítem representa una afirmación referida a pensamientos, sentimientos, actitudes o comportamientos durante la práctica del arbitraje. El formato de respuesta es tipo Likert (5= Casi siempre, $4=\mathrm{A}$ menudo, $3=\mathrm{A}$ veces, $2=$ Rara vez, $1=$ Casi nunca), y determinados ítems $(1,2,3,9$, $10,14,15,16,20,23,24,30,31,33$ y 38$)$ se puntúan de forma inversa, $(1=$ Casi siempre a $5=$ Casi nunca). Las subescales evaluadas son: Autoconfianza (AC): grado de convicción que tiene el árbitro para la consecución de las metas propuestas, mediante sus propias habilidades, estando determinada por la experiencia, los estados fisiológicos y emocionales, y se relaciona con el concepto de autoeficacia (Bandura, 1977); Control de Afrontamiento Negativo (CAN): capacidad de enfrentarse a las continuas situaciones adversas que se presentan (Csikszentmihalyi, 1992). En árbitros, pueden ser lances durante los partidos, entrenamientos o exámenes, contando, además, con que el árbitro 
tiene que hacer frente a la mayoría de situaciones de forma individual; Control Atencional (CAT): control de la concentración y capacidad para atender a la gran variedad de estímulos y situaciones que se presentan. En una variable clave en la toma de decisiones que permite mantener un estado de alerta, focalizado en los eventos destacados e ignorando los irrelevantes (Hernández-Mendo \& Ramos, 1996; Dosil \& Caracuel, 2003); Control Visuo-Imaginativo (CVI): capacidad de controlar las experiencias sensoriales y perceptivas en ausencia de estimulaciones externas y poder imaginar situaciones posibles en la toma de decisiones (Hernández-Mendo, 2002); Nivel Motivacional (NM): grado de atracción del árbitro hacia su actividad, y control de la conducta del árbitro ante ciertos procesos que activan, orientan, o lo dirigen hacia un determinado objetivo (Albo-Lucas \& Núñez-Alonso, 2003); Control de Afrontamiento Positivo (CAP): capacidad para lograr una mejor disposición a través de las emociones y estados positivos. Cuando el árbitro se enfrenta a situaciones deportivas favorables o adversas, tiene que ser capaz de mantener estables su conducta y sus actividades cognitivas para poder afrontarlas en un estado óptimo (Csikszentmihalyi, 1992); Control Actitudinal (CACT): disposición hacia su actividad, y dominio sobre acciones, conductas, o diferentes personas, así como su reacción ante ellas (Hernández-Mendo \& Morales-Sánchez, 2000).

\section{Procedimiento}

Se adaptó el contenido del IPED original modificando los ítems con términos como árbitro o partido para una mejor identificación y comprensión. A continuación, especialistas en arbitraje (árbitros de Tercera y Segunda División B de fútbol, y delegados informadores del Comité Técnico de Árbitros), y expertos en psicología del deporte, evaluaron el contenido, la adecuación sintáctica y semántica, y la conveniencia de inclusión o exclusión de cada uno de los ítems respecto al cuestionario y al constructo que miden. Sus valoraciones hicieron referencia a una fácil comprensión, relacionado con aspectos arbitrales a tener en cuenta para los partidos, entrenamientos o pruebas, así como para afrontar los problemas ocasionados por la frustración ante la no consecución de objetivos o gestionar los éxitos. Tras su valoración, se realizaron las modificaciones pertinentes y se obtuvo la versión final del inventario.

El cuestionario fue cumplimentado por la mayoría de los colegiados de forma presencial durante reuniones técnicas, previa explicación sobre el instrumento, objetivos de estudio y el tratamiento de confidencialidad que recibirían los datos, firmando el consentimiento. La muestra restante cumplimentó el cuestionario a través de la plataforma Google Drive, firmando consentimiento previo por correo electrónico.

\section{Análisis de datos}

En la validación del cuestionario se emplearon los paquetes estadísticos SPSS v.15.0, LISREL 8.80 y PRELIS 2.80 (Jöresbog \& Sörbom, 2006), FACTOR (Lorenzo-Seva \& Ferrando, 2013), SAGT v1.0 (Hernández-Mendo, Blanco-Villaseñor, Pastrana, Morales-Sánchez, \& Ramos-Pérez, 2016), y SAS 9.2. (Schlotzhauer \& Littell, 1997).

Mediante Alfa de Cronbach $(\alpha)$ se midió la consistencia interna del cuestionario. La consistencia interna debe estar entre .70 y .95 , según el grado o nivel de consolidación que presente el instrumento. Valores superiores indican que la escala tiene más de 15-20 ítems o que existe redundancia de ítems (Campo-Arias \& Oviedo, 2008; Keszei et al., 2010). Por ello, para obtener otro coeficiente de consistencia interna adicional, se utilizó también Omega $(\omega)$ de McDonald (1970), el cual trabaja con las cargas factoriales y no depende del número de ítems.

Se realizó un Análisis Factorial Confirmatorio (AFC), utilizando el método de estimación de factores ULS (Unweighted Least Squares) (Jöresbog, 1977), ya que según Lloret-Segura, Ferreres-Traver, Hernández-Baeza, y TomásMarco (2014) funciona adecuadamente cuando se trabaja con muestras pequeñas como la disponible en el IPEDA, incluso cuando el número de variables es elevado, aunque es necesario considerar que no aplica a todos los indicadores independientemente del método de estimación (Hu \& Bentler, 1999). Es un método válido para la adaptación de un cuestionario como el IPEDA, ya que se conocen los 42 ítems que forman los siete constructos, con seis ítems por constructo, 
igual que el IPED original. El AFC se realizó con los programas LISREL 8.30 y PRELIS 2.30. A partir de los resultados del AFC, se obtuvieron la fiabilidad compuesta, las saturaciones factoriales, la validez convergente, la validez discriminante, los índices de ajuste NFI (Índice de Ajuste Normalizado), CFI (Índice de Ajuste Comparado), GFI (Índice de Bondad de Ajuste), y AGFI (Índice Ajustado de Bondad) y los de error RMR (Residuo Cuadrático Medio), SRMR (Residuo Cuadrático Medio Estandarizado) y RMSEA (Error de Aproximación Cuadrático Medio), al igual que en el estudio de replicación del IPED de Hernández-Mendo et al. (2014).

Posteriormente, se realizó un análisis de generalizabilidad para estimar el grado de generalización de un diseño de medida respecto a las condiciones de un valor teórico buscado, asumiendo que cualquier situación de medida posee infinitas fuentes de variación, denominadas facetas (en el IPEDA participantes, ítems y los constructos). Este análisis permite unificar las diferentes definiciones de fiabilidad, validez y precisión. Para su realización, se implementó previamente un análisis de componentes de varianza para observar si alguna de las facetas contribuye más al error, mediante el programa SAS 9.2, usando un procedimiento de mínimos cuadrados (Varcomp Type I) y un procedimiento de máxima verosimilitud (General Linear Model GLM). La suma de cuadrados obtenidas se utilizó con el programa SAGT v1.0 denominando como facetas a participantes, ítems y la interacción de ambas, para el cálculo de generalizabilidad y la estimación de los coeficientes $\mathrm{G}$ relativo (fiabilidad $e^{2}$ ) y absoluto (generalizabilidad $(\Phi)$ ), considerados aceptables cuanto más cercanos a 1.00 .

\section{Resultados}

En la Tabla 1 se muestran las puntuaciones medias, las desviaciones típicas, y la consistencia interna de las escalas del cuestionario. El promedio más bajo es el de control atencional, 24.49 (DT=3.331), siendo el más elevado en control de afrontamiento positivo ( $\mathrm{M}=25.78$; $\mathrm{DT}=3.050$ ). En cuanto a la fiabilidad, el alfa de Cronbach oscila entre $\alpha=0.64$ para control de afrontamiento negativo y $\alpha=0.80$ de control visual e imaginativo. Los valores de omega de McDonald oscilan entre $\omega=.65$ control de afrontamiento negativo y $\omega=.80$ para control visual.

Tabla 1. Medias, desviaciones típicas y fiabilidad de los factores del IPEDA

\begin{tabular}{|c|c|c|c|c|}
\hline & Media & D.T. & $\begin{array}{c}\text { Alfa de } \\
\text { Cronbach }\end{array}$ & $\begin{array}{l}\text { Omega de } \\
\text { McDonald }\end{array}$ \\
\hline $\begin{array}{l}\text { Autoconfianza } \\
\text { C. }\end{array}$ & 25.45 & 2.930 & .65 & .69 \\
\hline $\begin{array}{l}\text { Afrontamiento } \\
\text { Negativo }\end{array}$ & 24.76 & 3.160 & .64 & .65 \\
\hline $\begin{array}{l}\text { Control } \\
\text { Atencional }\end{array}$ & 24.49 & 3.331 & .69 & .73 \\
\hline $\begin{array}{l}\text { Control Visual } \\
\text { e Imaginativo }\end{array}$ & 20.93 & 4.727 & .80 & .80 \\
\hline $\begin{array}{l}\text { Nivel } \\
\text { Motivacional } \\
\text { C. }\end{array}$ & 24.66 & 3.658 & .72 & .75 \\
\hline $\begin{array}{l}\text { Afrontamiento } \\
\text { Positivo }\end{array}$ & 25.78 & 3.050 & .68 & .69 \\
\hline $\begin{array}{l}\text { Control } \\
\text { Actitudinal }\end{array}$ & 24.89 & 3.145 & .73 & .73 \\
\hline
\end{tabular}

Los resultados de los índices de ajuste se muestran en la Tabla 2 . El valor de $\chi^{2} / \mathrm{df}=2.04$ se encuentra en el rango entre uno y tres, como propone Jöreskog (1970) para considerar un modelo aceptable. Además, se obtiene 1.00 en NFI, 1.00 en CFI, .92 en GFI y .91 en AGFI, mientras que para los índices de error se obtuvieron .083 en RMR, .083 en SRMR, .078 en RMSEA (IC90\%=.072-.083; $p<.001)$ Los resultados cercanos a 1.00 en ajuste y en el límite de .80 en error, cumplen con las recomendaciones de Arias (2008), Hu y Bentler (1999) y Schreiber, Nora, Stage, Barlow y King (2006).

En la Tabla 3 se muestran los resultados del cálculo de los índices de fiabilidad y validez a través del AFC para cada uno de los diferentes factores, así como los correspondientes ítems que componen cada factor, resultando satisfactoria la fiabilidad compuesta, con valores mayores de .60 . También la validez convergente, donde los 42 ítems del IPEDA son estadísticamente significativos, con un valor por encima de |1.96|, representando el constructo en el que saturan. Las saturaciones factoriales son superiores a .30, considerados aceptables, a excepción de los ítems 22 con $.21,23$ con .10 y 10 con .28 , éste último muy cercano al valor aceptable. 
Tabla 2. Índices de ajuste y error

\begin{tabular}{lccccccccc}
\hline$\chi^{2}$ & $\mathrm{gl}$ & RMSEA & IC90\% RMSEA & RMR & SRMR & NFI & CFI & GFI & AGFI \\
\hline 1631.38 & 798 & .078 & $.072-.083$ & .083 & .083 & 1.00 & 1.00 & .92 & .91 \\
\hline
\end{tabular}

Tabla 3. Índices de Fiabilidad y Validez

\begin{tabular}{|c|c|c|c|c|c|c|c|}
\hline Factor & Ítem & $\begin{array}{c}\text { Peso } \\
\text { factorial }\end{array}$ & $\begin{array}{l}\text { Fiabilidad } \\
\text { compuesta }\end{array}$ & $\begin{array}{c}\text { Varianza Media } \\
\text { extractada }\end{array}$ & $\begin{array}{c}\text { Validez } \\
\text { Convergente }\end{array}$ & \multicolumn{2}{|c|}{ Validez Discriminante } \\
\hline \multirow{6}{*}{ Autoconfianza } & 1 & .45 & \multirow{6}{*}{.68} & \multirow{6}{*}{.28} & 16.44 & $.79>$ & .25 \\
\hline & 8 & .64 & & & 21.60 & $.59>$ & .32 \\
\hline & 15 & .54 & & & 19.24 & $.32<$ & .38 \\
\hline & 22 & .21 & & & 7.50 & $.59>$ & .34 \\
\hline & 29 & .65 & & & 21.38 & $1.06>$ & .27 \\
\hline & 36 & .57 & & & 19.65 & $.96>$ & .31 \\
\hline & 2 & .51 & & \multirow{6}{*}{.25} & 14.73 & & \\
\hline Control de & 9 & .46 & \multirow{5}{*}{.63} & & 13.55 & $.83>$ & .32 \\
\hline Afrontamiento & 16 & .54 & & & 15.45 & $.21<$ & .38 \\
\hline \multirow[t]{3}{*}{ Negativo } & 23 & .10 & & & 3.43 & $.22<$ & .34 \\
\hline & 30 & .61 & & & 18.81 & $.79>$ & .27 \\
\hline & 37 & .57 & & & 15.90 & $.72>$ & .31 \\
\hline \multirow{6}{*}{$\begin{array}{l}\text { Control } \\
\text { Atencional }\end{array}$} & 3 & .61 & \multirow{6}{*}{.72} & \multirow{6}{*}{.32} & 17.97 & & \\
\hline & 10 & .28 & & & 09.31 & $.20<$ & .38 \\
\hline & 17 & .61 & & & 18.54 & $.36>$ & .34 \\
\hline & 24 & .55 & & & 17.02 & $.69>$ & .27 \\
\hline & 31 & .38 & & & 12.44 & $.76>$ & .31 \\
\hline & 38 & .81 & & & 22.24 & & \\
\hline \multirow{6}{*}{$\begin{array}{l}\text { Control } \\
\text { Visuo- } \\
\text { Imaginativo }\end{array}$} & 4 & .52 & \multirow{6}{*}{.78} & \multirow{6}{*}{.38} & 15.01 & & \\
\hline & 11 & .48 & & & 14.34 & & \\
\hline & 18 & .69 & & & 2.62 & $.45>$ & .34 \\
\hline & 25 & .60 & & & 16.53 & $.44>$ & .27 \\
\hline & 32 & .59 & & & 16.89 & $.45>$ & .31 \\
\hline & 39 & .78 & & & 21.77 & & \\
\hline \multirow{6}{*}{$\begin{array}{l}\text { Nivel } \\
\text { Motivacional }\end{array}$} & 5 & .75 & \multirow{6}{*}{.75} & \multirow{6}{*}{.39} & 21.36 & & \\
\hline & 12 & .67 & & & 19.33 & & \\
\hline & 19 & .58 & & & 17.73 & $.98>$ & .27 \\
\hline & 26 & .57 & & & 17.70 & $.66>$ & .31 \\
\hline & 33 & .39 & & & 12.84 & & \\
\hline & 40 & .45 & & & 14.48 & & \\
\hline \multirow{6}{*}{$\begin{array}{l}\text { Control de } \\
\text { Afrontamiento } \\
\text { Positivo }\end{array}$} & 6 & .43 & \multirow{6}{*}{.69} & \multirow{6}{*}{.27} & 16.07 & & \\
\hline & 13 & .56 & & & 18.65 & & \\
\hline & 20 & .55 & & & 18.86 & & \\
\hline & 27 & .60 & & & 19.78 & $1.08>$ & .31 \\
\hline & 34 & .40 & & & 13.52 & & \\
\hline & 41 & .57 & & & 19.08 & & \\
\hline \multirow{6}{*}{$\begin{array}{l}\text { Control } \\
\text { Actitudinal }\end{array}$} & 7 & .57 & \multirow{6}{*}{.73} & \multirow{6}{*}{.31} & 17.98 & & \\
\hline & 14 & .46 & & & 16.11 & & \\
\hline & 21 & .56 & & & 17.83 & & \\
\hline & 28 & .57 & & & 17.42 & & \\
\hline & 35 & .62 & & & 2.36 & & \\
\hline & 42 & .57 & & & 18.59 & & \\
\hline
\end{tabular}

Para obtener la validez discriminante, la varianza media extractada de cada uno de ellos debe ser superior al cuadrado de la correlación entre ellas. Únicamente son superiores las saturaciones del constructo Control Visual con los constructos Autoconfianza (.32<.38), Control de Afrontamiento Negativo $(.21<.81)$ y Capacidad Atencional $(.20<.38)$, y el constructo Nivel Motivacional con el constructo Control de Afrontamiento Negativo (.22<.34).

Seguidamente, como se puede observar en la Tabla 4, el análisis de componentes de varianza, realizado para identificar las fuentes de variación o facetas, arroja como resultados una suma de cuadrados de 7024.989 (1367.727 [P], 1130.087 [I] y $4527.175[\mathrm{P}][\mathrm{I}])$ y una media cuadrática de .961 .

Además, el R-cuadrado es de 1.000 , en tres facetas (participantes [P], ítems [I] y su interacción $[\mathrm{P}][\mathrm{I}])$. A partir de estos resultados se realizó el análisis de generalizabilidad para obtener los indicadores de fiabilidad $\left(e^{2}\right)$ y de generalizabilidad $(\Phi)$, mostrando resultados satisfactorios, con .977 en Coeficiente $G$ Relativo $\left(e^{2}\right)$ con un Error Relativo de .004 y .971 en Coeficiente $G$ Absoluto $(\Phi)$, con un error Absoluto de .005. Además, se explica el 100\% de la varianza dividida en $17.912 \%$ [P], $16.018 \%$ [I] y $66.07 \%[P][I])$. 
Tabla 4. Análisis de generalizabilidad y porcentajes de varianza asociada del modelo $y=[\mathrm{P}][\mathrm{I}]$

\begin{tabular}{lcccccccc}
\hline $\begin{array}{c}\text { Fuentes de } \\
\text { variación }\end{array}$ & $\begin{array}{c}\text { Suma de } \\
\text { cuadrados }\end{array}$ & $\begin{array}{c}\text { Grado de } \\
\text { libertad }\end{array}$ & $\begin{array}{c}\text { Cuadrado } \\
\text { medio }\end{array}$ & Aleatorio & Mixtos & Corregidos & $\%$ & $\begin{array}{c}\text { Error } \\
\text { estándar }\end{array}$ \\
\hline$[\mathrm{P}]$ & 1367.727 & 173 & 7.906 & .173 & .173 & .173 & 17.912 & .02 \\
{$[\mathrm{I}]$} & 113.087 & 41 & 27.563 & .155 & .155 & .155 & 16.018 & .034 \\
{$[\mathrm{P}][\mathrm{I}]$} & 4527.175 & 7093 & .638 & .638 & .638 & .638 & 66.070 & .011 \\
\hline
\end{tabular}

Nota. $[\mathrm{P}]=$ Participantes; [I]=Ítems

\section{Discusión}

La evaluación del ajuste de un modelo es un proceso relativo y, por lo tanto, es más adecuado evaluar conjuntamente diversos tipos de medida para valorar la aceptabilidad de un modelo. El índice de ajuste por excelencia en los modelos AFC es $\chi^{2}$, siendo aceptable cuando el nivel de significación asociado de $p$ sea superior a .05 , como se obtiene en este estudio. También, se puede considerar fiable la medida a partir de los valores resultantes en fiabilidad compuesta, todos superiores a .60. Las saturaciones factoriales, por su parte, se consideran aceptables al estar su valor por encima de .30. En cuanto a la validez convergente, proporciona evidencia de la validez de los indicadores utilizados para medir los constructos con valores superiores a |1.96| (Arias, 2008).

Del mismo modo, se han obtenido valores por encima de 0.90 en los índices de ajuste NFI, CFI, GFI y AGFI (Arias, 2008; Hu \& Bentler, 1999), e incluso superiores a .95 en CFI y NFI como proponen Schreiber et al. (2006) y Schreiber (2017), pudiendo ser considerados aceptables. Al mismo tiempo, se obtienen valores adecuados, en el límite de .08, en los índices de error RMR, SRMR y RMSEA (Browne \& Cudeck, 1993; Schreiber et al., 2006).

Respecto a los estudios previos que han aportado información sobre las propiedades del IPED (Hernández-Mendo, 2006; HernándezMendo et al., 2014; Raimundi et al., 2016), se obtienen resultados muy similares en los índices de ajuste siendo el GFI y el AGFI superiores al del estudio de Raimundi et al. (2016) (GFI=.900; AGFI=.890) e inferiores al de Hernández-Mendo et al. (2014) (GFI=.980; AGFI=.960), mientras que el NFI fue superior al del estudio de HernándezMendo et al. (2014) (NFI=.920) e inferior al de Raimundi et al. (2016) (NFI=1.010). El CFI es superior al de Hernández-Mendo et al. (2014)
(CFI=0.940), e igual que el de Raimundi et al. (2016) (CFI=1.000). En Alfa de Cronbach, el IPEDA es superior en todos los factores al estudio original del IPED (Hernández-Mendo, 2006), y en control visual, nivel motivacional y control actitudinal al de Raimundi et al. (2016). Por su parte, en fiabilidad compuesta, control visual y nivel motivacional del IPEDA son superiores respecto al estudio de Hernández-Mendo (2006), control atencional, nivel motivacional y control actitudinal del estudio de Hernández-Mendo et al. (2014), y control visual, nivel motivacional y control actitudinal de Raimundi et al. (2016). Únicamente se obtuvieron inferiores resultados en índices de error, pero siendo en su conjunto aceptables para la utilización del instrumento.

Señalar asimismo como los resultados del análisis de componentes de varianza para el modelo $y=$ participantes*ítem muestran que es significativo, y que explica el $100 \%$ de la varianza. Además, las dos estrategias de análisis permitieron determinar que los errores residuales son iguales para ambos procedimientos, y por tanto, se asume que la muestra es lineal, normal y homocedástica (Hemmerle \& Hartley, 1973; Searle, Casella, \& McCulloch, 1992). Los resultados del análisis de generalizabilidad indican niveles de fiabilidad y generalizabilidad del IPEDA altos. Estos datos son aceptables de acuerdo con los trabajos de Cuba-Dorado, GarcíaGarcía, y Hernández-Mendo (2015), y el de García-García et al. (2013).

Pero al mismo tiempo, debemos señalar que el estudio presenta limitaciones en cuanto al tamaño de la muestra y su selección local y no aleatoria, ya que 174 participantes no es un número elevado. Es necesario tener presente que los índices de bondad de ajuste son más confiables con tamaños de muestra superiores a 250 participantes ( $\mathrm{Hu} \&$ Bentler, 1999). Prueba de ello son los resultados obtenidos de 1.00 en CFI y NFI, o las saturaciones factoriales inferiores a 0.30 en tres de los 42 
ítems. Por ello, futuros trabajos deben estudiar a través del IPEDA las características psicológicas de diferentes colectivos arbitrales, no solo de fútbol. El análisis de muestras mayores y de diferentes disciplinas permitiría obtener más datos que contribuirían a conocer en mayor profundidad las propiedades del cuestionario, y además poder realizar comparaciones entre deportes y entre niveles de rendimiento.

En el arbitraje deportivo, diversas variables como la percepción subjetiva del juego, la presión ejercida por el público, los jugadores y los propios entrenadores o el efecto de diferentes estresores relacionados con el entorno más próximo al árbitro, ejercen un efecto directo sobre su bienestar psicológico (Pedrosa \& García-Cueto, 2016). Resulta indudable que la práctica del arbitraje se encuentra asociada a elevadas exigencias físicas y psicológicas, en muchos casos similares a las de cualquier deportista de alto nivel (Matkovic, Rupcic, \& Knjaz, 2014). Los árbitros son deportistas y parte esencial del espectáculo deportivo. $\mathrm{Al}$ analizar las puntuaciones obtenidas por los árbitros de este estudio en cada una de las escalas, en comparación con las puntuaciones obtenidas en el cuestionario IPED por diferentes muestras deportivas (Álvarez et al., 2014; Berengüí et al., 2011, 2013; Gómez-López et al., 2013; Hernández-Mendo, 2006; HernándezMendo et al., 2014; Izquierdo et al., 2006; LópezCazorla et al., 2015; López-Gullón et al., 2011, 2012; Raimundi et al., 2016), se observa como los árbitros obtienen las mayores puntuaciones en autoconfianza, control del afrontamiento negativo, control atencional y control del afrontamiento positivo. También obtienen puntuaciones mayores que el resto de muestras en control actitudinal, a excepción de luchadores de élite (López-Gullón et al., 2011). En nivel motivacional, los árbitros presentan puntuaciones inferiores a seis muestras y superiores a las de siete estudios. Las puntuaciones obtenidas en esos factores, muestran como los árbitros estudiados poseen buenas habilidades psicológicas para afrontar su ejecución en el deporte.

Para concluir, debemos advertir la importancia a nivel aplicado del IPEDA. El cuestionario permite evaluar las características psicológicas de los árbitros, para obtener así otro indicador fundamental del rendimiento deportivo, e identificar un perfil con los puntos fuertes y aspectos necesarios de mejora de los colegiados. Esa información debe servir para que los diferentes profesionales encargados de la formación y mejora del colectivo arbitral dispongan de datos relevantes que contribuyan a diseñar óptimos planes de preparación, y que permitan la adecuada programación del entrenamiento psicológico de acuerdo a las necesidades individuales de cada árbitro.

\section{Referencias}

Albo-Lucas, J. M., \& Núñez-Alonso, J. L. (2003). Motivación y deporte. En A. Hernández Mendo, Psicología del Deporte (Vol.1): Fundamentos 2 (pp. 84-104). Buenos Aires: Tulio Guterman.

Álvarez, O., Estevan, I., Falcó, C., HernándezMendo, A., \& Castillo, I. (2014). Perfil de habilidades psicológicas en taekwondistas universitarios y su relación con el éxito en competición. Cuadernos de Psicología del Deporte, 14(3), 13-20.

Arias, B. (2008). Desarrollo de un ejemplo de análisis factorial confirmatorio con LISREL, AMOS y SAS. En M.A. Verdugo, M. Crespo, M. Badía y B. Arias (Coords.), Metodología en la investigación sobre discapacidad. Introducción al uso de las ecuaciones estructurales (pp. 75-120). Salamanca: Publicaciones del INICO.

Bandura, A. (1977). Self-efficacy: Towards a unifying theory of behavioral change. Psychological Review, 84, 191-215. doi:10.1037/0033-295X.84.2.191

Berengüí, R., Garcés de Los Fayos, E. J., \& Hidalgo-Montesinos, M. D. (2013). Características psicológicas asociadas a la incidencia de lesiones en deportistas de modalidades individuales. Anales de Psicología, 29(3), 674-684. doi:10.6018/analesps.29.3.175811

Berengüí, R., López-Gullón, J. M., Garcés de los Fayos, E. J., \& Almarcha, J. (2011). Factores psicológicos y lesiones deportivas en lucha olímpica y taekwondo. E-balonmano.com: Revista de Ciencias del Deporte, 7(Supl.), 9198. 
Browne, M. W., \& Cudeck, R. (1993). Alternative ways of assessing model fit. En K. A. Bollen \& J. S. Long (Eds.), Testing structural equation models (pp. 136-162). Newbury Park: Sage.

Bueno, J. M., \& Da Silva, A. I. (2015). Personal trainer e o treinamento específico para o árbitro de futebol. RBPFEX-Revista Brasileira de Prescrição e Fisiologia do Exercício, 9(52), 216-227.

Campo-Arias, A., \& Oviedo, H. C. (2008). Propiedades psicométricas de una escala: la consistencia interna. Revista de Salud Pública, 10, 831-839.

Campos-Salinas, J. A. (2017). Factores determinantes del arbitraje en fútbol: Análisis de los árbitros de la Región de Murcia. Tesis Doctoral. Universidad Católica de Murcia, Murcia, España.

Casajús, J. A., \& González-Aguero, A. (2015). Body Composition Evolution in Elite Football Referees; an Eleven-years Retrospective Study. International Journal of Sports Medicine, 36(7), 550-553. doi:10.1055/s-0034-1398582

Csikszentmihalyi, M. (1992). Flow. The psychology of Happiness. London: Rider.

Cuba-Dorado, A., García-García, O., \& Hernández-Mendo, A. (2015). Análisis de la capacidad explicativa de las pruebas de detención de talento en el rendimiento en competición de jóvenes triatletas. Cuadernos de Psicología del Deporte, 15(2), 105-112.

Dosil, J., \& Caracuel, J. C. (2003). Psicología aplicada al deporte. En J. Dosil (Ed.), Ciencias de la actividad física y el deporte. Madrid: Síntesis.

García-García, O., Morales-Sánchez, V., Hernández-Mendo, A., \& Serrano-Gómez, V. (2013). Aplicación de la teoría generalizabilidad a un análisis de tensiomiografía en ciclistas profesionales de ruta. Revista de Psicología del Deporte, 22(1), 53-60.

Giske, R., Haugen, T., \& Johansen, B. T. (2016). Training, mental preparation and unmediated practice among soccer referees: An analysis of elite and subelite referees reported practice. International Journal of Applied Sports Sciences, 28(1), 31-41. doi:10.24985/ijass.2016.28.1.31

Gómez-López, M. Granero-Gallegos, A., \& Isorna, M. (2013). Análisis de los factores psicológicos que afectan a los piragüistas en el alto rendimiento. Revista Iberoamericana de Diagnóstico y Evaluación Psicológica - e Avaliação Psicológica, 35(1), 57-76.

González-Oya, J. L., \& Dosil, J. (2004). Características psicológicas de los árbitros de futbol de la comunidad autónoma gallega. Cuadernos de Psicología del Deporte, 4(1 y 2), 53-66.

González-Oya, J. L., \& Díaz, J. (2007). La Psicología del árbitro de fútbol. A Coruña: Toxosoutos.

Guillén, F., \& Jiménez H. (2001). Características deseables en el arbitraje y juicio deportivo. Revista de Psicología del Deporte, 10(1), 2334.

Hancock, D. J., Rix-Lièvre, G., \& Côté, J. (2015). Citation network analysis of research on sport officials: A lack of interconnectivity. International Review of Sport and Exercise Psychology, 8(1), 95-105. doi: 10.1080/1750984X.2015.1022202

Hemmerle, W., \& Hartley, H. (1973). Computing maximum likelihood estimates for the mixed AOV Model using the w-transformation. Technometrics, 15, 819-831. doi:10.1080/00401706.1973.10489115

Hernández-Mendo, A. (2002). Imágenes mentales y deporte. En J. Dosil, Psicología y Rendimiento Deportivo (pp. 83-102). Ourense: Gersam.

Hernández-Mendo, A. (2006). Un cuestionario para la evaluación psicológica de la ejecución deportiva: Estudio complementario entre TCT y TRI. Revista de Psicología del Deporte, 15(1), 71-93.

Hernández-Mendo, A., Blanco-Villaseñor, A., Pastrana, J. L., Morales-Sánchez, V., \& Ramos-Pérez, F. J. (2016). SAGT: Aplicación informática para análisis de generalizabilidad. Revista Iberoamericana de Psicología del Ejercicio y el Deporte, 11(1), 77-89.

Hernández-Mendo, A., \& Morales-Sánchez, V. (2000). La actitud en la práctica deportiva: concepto. Lecturas: EF y Deportes. Revista Digital, 18. 
Hernández-Mendo, A., Morales-Sánchez, V., \& Peñalver, I. (2014). Replicación de las propiedades psicométricas del inventario psicológico de ejecución deportiva. Revista de Psicología del Deporte, 23(2), 311-324.

Hernández-Mendo, A., \& Ramos, R. (1996). Introducción a la informática aplicada a la psicología del deporte. Herramientas informáticas de uso en las ciencias del deporte. Madrid: Editorial Ra-Ma.

Hu, L. T., \& Bentler, P. M. (1999). Cutoff criteria for fit indexes in covariance structure analysis: Conventional criteria versus new alternatives. Structural Equation Modeling, 6(1), 1-55. doi: 10.1080/10705519909540118

Izquierdo, J., Ruiz, J., Navarro, R., Mendoza, M., Rodríguez, M., \& Brito, M. (2006). Habilidades psicológicas de ejecución deportiva en jugadores de futbol de Gran Canaria. Canarias Médica y Quirúrgica, 4(10), 39-48.

Jöreskog, K. G. (1970). A general method for analysis of covariance structures. Biometrika, 57(2), 239-251.

Jöresbog, K. G. (1977). Factor analysis by leastsquares and maximum-likelihood methods. En K. Enslein, A. Ralston y H.S. Wilf (Eds.), Statistical methods for digital computers, Vol. 3. New York: Wiley.

Jöresbog, K. G., \& Sörbom, D. (2006). LISREL 8.80: A guide to the program and applications. Chicago, IL: SPSS, Inc.

Kaiser, H. F. (1970). A second generation little jiffy. Psychometrika, 35, 401-415. doi: 10.1007/bf02291817

Keszei, A. P., Novak, M., \& Streiner, D. L. (2010). Introduction to health measurement scales. Journal of Psychosomatic Research, 68(4), 319-323.

Lloret-Segura, S., Ferreres-Traver, A., HernándezBaeza, A., \& Tomás-Marco, I. (2014). El análisis factorial exploratorio de los ítems: Una guía práctica, revisada y actualizada. Anales de Psicología,30(3), 1151-1169. doi:10.6018/analesps.30.3.199361

Loehr, J. E. (1982). Athletic excellence: Mental toughness training for sports. New York: The Stephen Greene Press.
Loehr, J. E. (1986). Mental toughness training for sports: Achieving athletic excellence. Lexington: Stephen Greene Press.

Loehr, J. E. (1995). The new toughness training for sports: Mental, emotional, and physical conditioning. Nueva York: A Plume Book.

López-Cazorla, R., Hernández-Mendo, A., Reigal R. E., \& Morales-Sánchez, V. (2015). Relaciones entre el autoconcepto y el perfil psicológico deportivo en triatletas. Cuadernos de Psicología del Deporte, 15(2), 95-102.

López-Gullón, J. M., García-Pallares, J., Berengüí, R., Martínez-Moreno, A., MoralesBaños, V., Torres-Bonete, M. D., \& Díaz, A. (2011). Factores físicos y psicológicos predictores del éxito en lucha olímpica. Revista de Psicología del Deporte, 20(2), 573588.

López-Gullón, J. M., Torres, M. D., Berengüí, R., Díaz, A., Martínez, A., Morales-Baños, V., \& García-Pallares, J. (2012). Rendimiento físico y psicológico en lucha olímpica: Predictores del éxito en lucha femenina. Anales de Psicología, 28(1), 215-222.

Lorenzo-Seva, U., \& Ferrando, P. J. (2013). FACTOR 9.2: A comprehensive program for fitting exploratory and semiconfirmatory factor analysis and IRT models. Applied Psychological Measurement, 37-6, 497-498. doi:10.1177/0146621613487794

Matkovic, A., Rupcic, T., \& Knjaz, D. (2014). Physiological load of referees during basketball games. Kinesiology, 14(2), 258265.

McDonald, R. P. (1970). The theoretical foundations of principal factor analysis, canonical factor analysis, and alpha factor analysis. British Journal of Mathematical and Statistical Psychology, 23(1), 1-21.

Pedrosa, I., \& García-Cueto, E. (2016). Síndrome de Burnout en Árbitros de Élite: La Liga de Fútbol Profesional Española (LFP) a Estudio. Revista Iberoamericana de Diagnóstico y Evaluación - e Avaliação Psicológica, 42(2), 59-68. doi:10.21865/RIDEP42_59

Raimundi, M. J., Reigal, R., \& Hernández-Mendo, A. (2016). Adaptación argentina del Inventario Psicológico de Ejecución Deportiva (IPED): Validez, fiabilidad y 
precisión. Cuadernos de Psicología del Deporte, 16(1), 211-222.

Schlotzhauer, S. D., \& Littell, R. C. (1997). SAS Sistem for Elementary Statical Analysis. Cary, NC: SAS Institute Inc.

Schreiber, J. B. (2017). Update to core reporting practices in structural equation modeling. Research in Social and Administrative Pharmacy, 13(3), 634-643.

Schreiber, J. B., Nora, A., Stage, F. K., Barlow, E. A., \& King, J. (2006). Reporting structural equation modeling and confirmatory factor analysis results: A review. The Journal of Educational Research, 99(6), 323-337. doi:10.1016/j.sapharm.2016.06.006

Searle, S., Casella, G., \& McCulloch, C. (1992). Variance components. New York: John Wiley y Sons. 
ANEXO.

Ítems del Inventario Psicológico de Ejecución Deportiva para Árbitros (IPEDA)

1. Me veo más como un mal árbitro que como un buen árbitro durante los partidos.

2. Me enfado y frustro durante los partidos.

3. Llego a distraerme y perder mi concentración durante los partidos.

4. Antes de los partidos, me imagino a mí mismo ejecutando mis acciones y rindiendo perfectamente.

5. Estoy muy motivado para dar lo mejor de mí en los partidos.

6. Puedo mantener emociones positivas durante los partidos.

7. Durante los partidos pienso positivamente.

8. Creo en mí mismo como árbitro.

9. Me pongo nervioso durante los partidos.

10. En los momentos críticos de los partidos me da la impresión de que mi cabeza va muy deprisa.

11. Practico mentalmente mis habilidades físicas.

12. Trabajo y entreno duro gracias a los objetivos que yo me he fijado como árbitro.

13. Disfruto durante los partidos, aunque me encuentre con la presencia de dificultades.

14. Durante los partidos mantengo auto conversaciones de carácter negativo.

15. Pierdo mi confianza fácilmente

16. Los errores durante los partidos me hacen sentir y pensar negativamente.

17. Puedo controlar rápidamente mis emociones y recuperar la concentración.

18. Para mí es fácil pensar fotográficamente (en imágenes) acerca de jugadas y decisiones.

19. No necesito que me empujen a entrenar duro y arbitrar con intensidad. Yo soy mi mejor elemento de motivación.

20. Cuando las cosas se vuelven contra mí durante los partidos, tiendo a desinflarme emocionalmente.

21. Empleo todo mi esfuerzo durante los partidos, pase lo que pase.

22. Puedo rendir por encima de mi talento y habilidades.

23. Durante los partidos siento que mis músculos se tensan y creo que no me van a responder.

24. Me distraigo durante los partidos.

25. Antes de los partidos, me visualizo superando situaciones difíciles y ejecutando acciones complejas.

26. Daría lo que fuera por desarrollar todo mi potencial y alcanzar la cumbre como árbitro.

27. Entreno y arbitro con una intensidad alta y positiva.

28. Controlando mi pensamiento soy capaz de transformar estados de humor negativos en positivos.

29. Soy un árbitro mentalmente tenaz.

30. Cuando arbitro, las situaciones incontrolables, como el viento, las trampas de los jugadores, o interferencias externas, me alteran y hacen que me derrumbe.

31. Durante los partidos pienso en errores pasados o decisiones desacertadas.

32. Durante los partidos utilizo imágenes que me ayudan a rendir mejor.

33. Estoy aburrido y quemado con el arbitraje.

34. Las situaciones difíciles para mí suponen un desafío y me inspiran.

35. Los informadores dirían de mí que tengo una buena actitud como árbitro.

36. La imagen que proyecto al exterior es de ser un luchador.

37. Puedo permanecer tranquilo durante los partidos pese a que aparezcan problemas perturbadores.

38. Mi concentración se rompe fácilmente.

39. Cuando me visualizo arbitrando o entrenando, puedo ver y sentir las cosas muy vivamente.

40. Al despertar por las mañanas me siento entusiasmado en relación con los entrenamientos y los partidos.

41. Practicar el arbitraje me aporta un sentido genuino de disfrute y realización.

42. Yo puedo convertir un problema deportivo (crisis) en una oportunidad. 